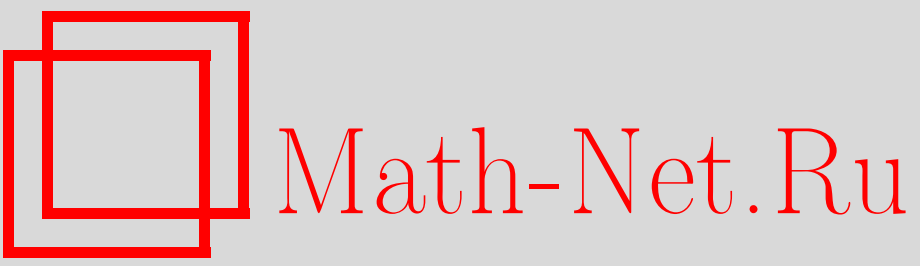

Аг. Х. Ханмамедов, Прямая и обратная задачи рассеяния для возмущенного разностного уравнения Хилла, Матем. сб., 2005, том 196, номер 10, 137-160

DOI: https://doi.org/10.4213/sm1429

Использование Общероссийского математического портала Math-Net.Ru подразумевает, что вы прочитали и согласны с пользовательским соглашением

http://www.mathnet.ru/rus/agreement

Параметры загрузки:

IP: 54.80 .97 .219

26 апреля 2023 г., 06:57:20 


\section{Прямая и обратная задачи рассеяния для возмущенного разностного уравнения Хилла}

\footnotetext{
Изучаются прямая и обратная задачи рассеяния для возмущенного уравнения Хилла $\left(\widehat{a}_{n-1}+a_{n-1}\right) y_{n-1}+\left(\widehat{b}_{n}+b_{n}\right) y_{n}+\left(\widehat{a}_{n}+a_{n}\right) y_{n+1}=\lambda y_{n}, n \in \mathbb{Z}$. По периодическим коэффициентам $\widehat{a}_{n}, \widehat{b}_{n}$ и по данным рассеяния восстанавливаются коэффициенты возмущения $a_{n}, b_{n}$.

Библиография: 21 название.
}

\section{Введение}

Некоторые физические явления можно моделировать разностными уравнениями второго порядка. Примерами являются колебание частиц в одномерных линейных цепочках [1], разностная аппроксимация дифференциальных уравнений [2] и т.д. В работах [2]-[7] изучалась обратная задача рассеяния для разностного уравнения Штурма-Лиувилля

$$
p_{n-1} y_{n-1}+q_{n} y_{n}+p_{n} y_{n+1}=\lambda y_{n}, \quad n=0, \pm 1, \ldots,
$$

в случае, когда коэффициенты $p_{n}, q_{n}$ сходятся на бесконечности. Однако классические рассуждения, использованные в [2]-[7], не позволяют исследовать обратную задачу для разностного уравнения Штурма-Лиувилля с расходящимися коэффициентами. Это связано в первую очередь с тем, что решения соответствующего стационарного уравнения, имеющие асимптотики на бесконечности, подвергаются сушественным изменениям (см. [8]). Последнее приводит к нарушению аддитивного свойства функции рассеяния. В то же время в связи с запросами теории нелинейных уравнений, интегрируемых методом обратной задачи рассеяния, возникает необходимость изучения таких задач. Кроме того, разностные аналоги некоторых непрерывных моделей таких, как одномерная система Дирака, описываются (см. [8]) разностными уравнениями Штурма-Лиувилля в случае, когда сходятся лишш специальные подпоследовательности коэффициентов $p_{n}, q_{n}$.

В настояшей работе исследуются прямая и обратная задачи рассеяния для возмушенного разностного уравнения Хилла

$$
\left(\widehat{a}_{n-1}+a_{n-1}\right) y_{n-1}+\left(\widehat{b}_{n}+b_{n}\right) y_{n}+\left(\widehat{a}_{n}+a_{n}\right) y_{n+1}=\lambda y_{n}, \quad n=0, \pm 1, \ldots,
$$

в котором вешественные коэффициенты $\widehat{a}_{n}, a_{n}, \widehat{b}_{n}, b_{n}$ удовлетворяют условиям

$$
\begin{array}{ll}
\widehat{a}_{n+N}=\widehat{a}_{n}>0, \quad \widehat{b}_{n+N}=\widehat{b}_{n}, \widehat{a}_{n}+a_{n}>0, & n=0, \pm 1, \pm 2, \ldots, \\
Q_{r}=\sum_{n=-\infty}^{\infty}|n|^{r}\left(\left|a_{n}\right|+\left|b_{n}\right|\right)<\infty, & r=1 \text { или } r=2,
\end{array}
$$


где $N \geqslant 2$ - натуральное число. Это охватывает тот случай, когда коэффициенты разностного уравнения Штурма-Лиувилля содержат $N$ сходящихся специальных подпоследовательностей. При $N=2$ эта задача рассматривалась в работах [9]-[11]. Однако решения обратных задач рассеяния в этих статьях нельзя считать удовлетворительными в силу отсутствия доказательства положительности некоторых выражений, связанных с коэффициентом возмушения $a_{n}$. Кроме того, при $\widehat{b}_{n}+b_{n} \equiv 0$ этот случай изучен в [12] для более широкого класса коэффициентов возмушения $a_{n}$.

В работе найдены условия на данные рассеяния, которые являются необходимыми $\left(\right.$ при $\left.Q_{2}<\infty\right)$ и достаточными для существования единственных коэффициентов $a_{n}, b_{n}$ с данньми характеристиками рассеяния и конечньм первым моментом $Q_{1}<\infty$.

Отметим, что уравнение (0.1) слу жит разностным аналогом возмущенного уравнения Хилла

$$
-y^{\prime \prime}+p(x) y+q(x) y=\lambda y, \quad p(x+1)=p(x), \quad x \in \mathbb{R},
$$

обратная задача рассеяния для которого исследовалась в работах [13], [14]. Как следует из [14], даже для примесных потенциалов $q(x)$ из класса

$$
\int_{-\infty}^{\infty}\left(1+x^{2}\right)|q(x)| d x<\infty
$$

обратную задачу не удается решить столь же эффективно, как обратную задачу в случае $p(x)=0$ (см. также $[15 ;$ гл. $3, \S 5])$.

Работа состоит из пяти параграфов. Первый параграф̆ посвящен изучению решений $Ф$ локе соответствуюшего невозмущенного (стационарного) уравнения. С их помощью построены так называемые “регуляризованные" решения Флоке. В $\S 2$ изложена (при $Q_{2}<\infty$ ) теория рассеяния для уравнения $(0.1)$, т.е. построены данные рассеяния, изучены их свойства. Здесь мы следуем схеме, предложенной и разработанной в статьях [3]-[7], где обратная задача решена при $N=1$. В $\S 3$ получены основные уравнения типа Марченко, позволяющие решить обратную задачу. С помощью этих уравнений найдены некоторые свойства функций рассеяния. При этом некоторые характерные свойства такие, как неаддитивность функций рассеяния, не позволяют дословно применить методики, использованные в [3]-[7]. Последние два параграфа посвящены решению обратной задачи, т.е. задаче восстановления коэффициентов $a_{n}, b_{n}$ по невозмушенным коэффициентам $\widehat{a}_{n}, \widehat{b}_{n}$ и по данным рассеяния. Наличие периодических коэффициентов приводит к тому, что решению невозмушенного уравнения соответствует некоторая система функций, образующая базис в некотором весовом пространстве. При решении обратной задачи мы пользуемся разложениями некоторых функций по такому базису. Исследование таких разложений требует ряда ухищрений. Это обстоятельство и отсутствие аддитивных свойств функции рассеяния вносят существенные трудности при применении традиционных подходов к решению обратной задачи. 


\section{§1. Исследование невозмущенного уравнения}

Через $\varphi_{n}=\varphi_{n}(\lambda)$ и $\theta_{n}=\theta_{n}(\lambda)$ обозначим решения невозмушенного уравнения

$$
\widehat{a}_{n-1} y_{n-1}+\widehat{b}_{n} y_{n}+\widehat{a}_{n} y_{n+1}=\lambda y_{n}, \quad n=0, \pm 1, \pm 2, \ldots,
$$

удовлетворяющие условиям $\varphi_{0}=\theta_{1}=0, \varphi_{1}=\theta_{0}=1$. Обозначим $\Delta(\lambda)=$ $2^{-1}\left[\varphi_{N+1}+\theta_{N}\right]$. Известно, что [16; гл. $\left.4, \S 4\right]$ корни многочлена $\Delta^{2}(\lambda)-1$ могут быть пронумерованы в невозрастающем порядке:

$$
\lambda_{1}>\lambda_{2} \geqslant \lambda_{3}>\lambda_{4}>\cdots>\lambda_{2 N-2} \geqslant \lambda_{2 N-1}>\lambda_{2 N} .
$$

При этом множество $\sigma=\{\lambda:|\Delta(\lambda)| \leqslant 1\}=\bigcup_{r=1}^{N} \sigma_{r}$, где $\sigma_{r}=\left[\lambda_{2 r}, \lambda_{2 r-1}\right], r=$ $1, \ldots, N$, является непрерывньм спектром оператора, порожденного левой частью уравнения (1.1) в пространстве $l^{2}(-\infty, \infty)$. Многочлен $\Delta(\lambda)$ монотонно убьвает от 1 до -1 на четных компонентах спектра $\sigma_{2 r}$ и возрастает от -1 до 1 на нечетных компонентах $\sigma_{2 r-1}$. Только отрезки $\left[\lambda_{2 r+1}, \lambda_{2 r}\right], r=1, \ldots, N-1$, которые мы назовем лакунами, могут вырождаться в точку, давая двукратно вырожденные корни. В каждой лакуне лежит [16], [17] по одному (простому) нулю многочленов $\varphi_{N}(\lambda), \Delta_{\lambda}(\lambda), \theta_{N+1}(\lambda)$.

Пусть $\Gamma$ - комплексная $\lambda$-плоскость с разрезами по отрезкам $\sigma_{r}, r=1,2, \ldots, N$. В плоскости $Г$ рассмотрим функцию

$$
z=z(\lambda)=\Delta(\lambda)+\sqrt{\Delta^{2}(\lambda)-1}
$$

выбирая регулярную ветвь радикала такую, что $\sqrt{\Delta^{2}(\lambda)-1}<0$ при $\lambda>\lambda_{1}$. Тогда [18] функция $z=z(\lambda)$ непрерьвна вплоть до границы $\partial Г$ и обладает свойствами

$$
\begin{gathered}
|z|<1 \text { при } \lambda \in \Gamma, \quad|z|=1 \text { при } \lambda \in \partial \Gamma, \\
z=\frac{A}{\lambda^{N}}+O\left(\frac{1}{\lambda^{N+1}}\right), \quad \lambda \rightarrow \infty, \quad A=\widehat{a}_{1} \widehat{a}_{2} \cdots \widehat{a}_{N} .
\end{gathered}
$$

Существует [18] число $r>0$ такое, что для любой точки $\lambda_{0} \in \sigma$ замкнутьй полукруг $T^{ \pm}=\left\{\lambda:\left|\lambda-\lambda_{0}\right| \leqslant r, \pm \operatorname{Im} \lambda \geqslant 0\right\}$ конформно отображается функцией $z=z(\lambda)$ на внутренность единичного круга $|z| \leqslant 1$.

Как следует из [8], [17], уравнение (1.1) имеет решения $\Psi_{n}^{ \pm}=\Psi_{n}^{ \pm}(\lambda)$, представимые в виде $\Psi_{n N+j}^{ \pm}=E_{j+1}^{ \pm} z^{ \pm n}, j=0,1, \ldots, N-1$, где функции $E_{j}=E_{j}(\lambda)$ определяются формулами

$$
\begin{aligned}
E_{1}^{ \pm} \equiv 1, \quad E_{m}^{ \pm}= & \chi^{-1}(1, N-1 ; \lambda)\left[\widehat{a}_{m-1} \widehat{a}_{m} \cdots \widehat{a}_{N-1} \chi(1, m-2 ; \lambda) z^{ \pm 1}\right. \\
& \left.+\widehat{a}_{0} \widehat{a}_{1} \cdots \widehat{a}_{m-2} \chi(m, N-1 ; \lambda)\right], \quad m=2, \ldots, N
\end{aligned}
$$

причем $\chi(1,0 ; \lambda)=\chi(N, N-1 ; \lambda) \equiv 1$,

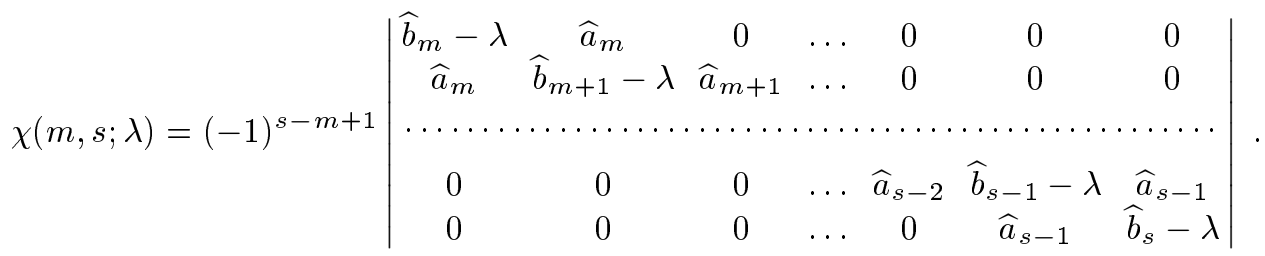


Так как для любой точки $\lambda \in \Gamma$ выполняются соотношения $\Psi_{n}^{ \pm}(\lambda) \in l^{2}(0, \pm \infty)$, то функции $\Psi_{n}^{ \pm}(\lambda)$ служат решениями $\Phi$ локе для уравнения (1.1)

$$
\Psi_{n}^{ \pm}(\lambda)=\theta_{n}(\lambda)+m^{ \pm}(\lambda) \varphi_{n}(\lambda), \quad \lambda \in \Gamma
$$

Учитывая, что $\Psi_{n}^{ \pm}(\lambda)=z^{ \pm 1}=\Delta(\lambda) \pm \sqrt{\Delta^{2}(\lambda)-1}$, имеем

$$
m^{ \pm}(\lambda)=\frac{\varphi_{N+1}-\theta_{N}}{2 \varphi_{N}} \pm \frac{\sqrt{\Delta^{2}(\lambda)-1}}{\varphi_{N}}
$$

откуда вытекает, что $m^{+}(\lambda) m^{-}(\lambda)=-\theta_{N+1} / \varphi_{N}$. В силу вышеупомянутых свойств функций $\varphi_{N}, \theta_{N+1}$ и последнего равенства в нулях многочлена $\varphi_{N}=$ $\varphi_{N}(\lambda)$ одно из решений $\Psi_{n}^{ \pm}(\lambda)$ регулярно, а другое имеет простой полюс. Более того, эти решения непрерывны вплоть до $\partial Г$ плоскости $\Gamma$.

Далее, как следует из $[16 ;$ гл. $4, \S 4]$, для производной $\Delta^{\prime}(\lambda)$ функции $\Delta(\lambda)$ справедлива формула

$$
2 \widehat{a}_{N} \Delta^{\prime}(\lambda)=\varphi_{N} \sum_{n=1}^{N}\left(\theta_{n}^{2}+\frac{\varphi_{N+1}-\theta_{N}}{\varphi_{N}} \varphi_{n} \theta_{n}-\frac{\theta_{N+1}}{\varphi_{N}} \varphi_{n}^{2}\right) .
$$

С другой стороны, в силу (1.4), (1.5) получаем

$$
\Psi_{n}^{+}(\lambda) \Psi_{n}^{-}(\lambda)=\theta_{n}^{2}+\frac{\varphi_{N+1}-\theta_{N}}{\varphi_{N}} \varphi_{n} \theta_{n}-\frac{\theta_{N+1}}{\varphi_{N}} \varphi_{n}^{2}
$$

Замечая, что $\chi(1, N-1 ; \lambda)=\widehat{a}_{1} \widehat{a}_{2} \cdots \widehat{a}_{N-1} \varphi_{N}(\lambda), \Psi_{n N+j}^{ \pm}=E_{j+1}^{ \pm} z^{ \pm n}$, имеем

$$
2 A \Delta^{\prime}(\lambda)=\chi(1, N-1 ; \lambda) \sum_{r=1}^{N} E_{r}^{+} E_{r}^{-}
$$

В дальнейшем нам понадобятся некоторые свойства решений Флоке. Пусть

$$
\rho_{0}(\lambda)=\frac{A \chi(1, N-1 ; \lambda)}{\left(z-z^{-1}\right)}
$$

Лемма 1.1. Имеет место формула

$$
\frac{1}{2 \pi i} \int_{\partial \Gamma} \rho_{0}(\lambda) \Psi_{n}^{+}(\lambda) \Psi_{m}^{-}(\lambda) d \lambda=\delta_{n m}, \quad n, m=0, \pm 1, \pm 2, \ldots
$$

әде $i-$ мнимая единица, $\delta_{n m}-$ символ Кронекера. 
ДокАЗАТЕЛЬСтво. Воспользовавшись теоремой о вычетах, находим, что

$$
\frac{1}{2 \pi i} \int_{\partial \Gamma} \rho_{0}(\lambda) \Psi_{n}^{+}(\lambda) \Psi_{m}^{-}(\lambda) d \lambda=\underset{\lambda=\infty}{\operatorname{res}} \rho_{0}(\lambda) \Psi_{n}^{+}(\lambda) \Psi_{m}^{-}(\lambda)
$$

Согласно (1.3) при $n \geqslant m$ верно соотношение

$$
\rho_{0}(\lambda) \Psi_{n}^{+}(\lambda) \Psi_{m}^{-}(\lambda)=-\frac{\delta_{n m}}{\lambda}+O\left(\frac{1}{\lambda^{2}}\right), \quad \lambda \rightarrow \infty
$$

откуда следует справедливость формулы (1.7) при $n \geqslant m$. Принимая во внимание, что $\overline{\rho_{0}(\lambda)}=-\rho_{0}(\lambda), \overline{\Psi_{n}^{+}(\lambda)}=\Psi_{n}^{-}(\lambda)$ при $\lambda \in \partial \Gamma$, получаем $(1.7)$ для всех $n$ и $m$.

Лемма доказана.

Обозначим $\widehat{\rho}(\lambda)=-i \rho_{0}(\lambda+i 0), \lambda \in \sigma$. Согласно определению функции $z=z(\lambda)$ выражение $i(-1)^{k-1}\left(z-z^{-1}\right)$ на верхнем берегу разреза $\sigma_{k}$ принимает неотрицательные значения. Кроме этого многочлен $\chi(1, N-1 ; \lambda)$, устремляясь к $+\infty$ при $\lambda \rightarrow+\infty$, имеет ровно один нуль между компонентами $\sigma_{k+1}$ и $\sigma_{k}$. Следовательно, во внутренних точках спектра $\sigma$ справедливо равенство $\widehat{\rho}(\lambda)>0$.

Введем в рассмотрение пространство $L_{2}\left(\partial \Gamma ; \rho_{0}(\lambda)\right)$ функций $f(\lambda)$, определенных на ӘГ и удовлетворяющих условиям

$$
f(\lambda-i 0)=\overline{f(\lambda+i 0)} \text { при п.в. } \quad \lambda \in \sigma,\left.\quad\left|\int_{\partial \Gamma} \rho_{0}(\lambda)\right| f(\lambda)\right|^{2} d \lambda \mid<\infty .
$$

Ясно, что $L_{2}\left(\partial \Gamma ; \rho_{0}(\lambda)\right)$ представляет собой евклидово пространство со скалярньм произведением

$$
\langle f, g\rangle=\frac{1}{2 \pi i} \int_{\partial \Gamma} \rho_{0}(\lambda) f(\lambda) \overline{g(\lambda)} d \lambda .
$$

Легко проверить, что это пространство есть сепарабельноегильбертово пространство; доказательство точно такое же, как и в случае обычного пространства $L_{2}$ (см. [19; гл. $7, \S 2])$.

ЛЕмма 1.2. Системь функиий $\left\{\Psi_{n}^{+}(\lambda)\right\}_{n=-\infty}^{\infty} u\left\{\Psi_{n}^{-}(\lambda)\right\}_{n=-\infty}^{\infty}$ являются базисами в $L_{2}\left(\partial \Gamma ; \rho_{0}(\lambda)\right)$.

ДоказАТЕльство. Достаточно показать, что в $L_{2}\left(\partial \Gamma ; \rho_{0}(\lambda)\right)$ не существует ненулевого элемента, ортогонального всем элементам системы $\left\{\Psi_{n}^{ \pm}(\lambda)\right\}_{n=-\infty}^{\infty}$. Пусть $f(\lambda) \in L_{2}\left(\partial \Gamma ; \rho_{0}(\lambda)\right)$ и

$$
\int_{\partial \Gamma} \rho_{0}(\lambda) f(\lambda) \overline{\Psi_{n}^{ \pm}(\lambda)} d \lambda=0, \quad n=0, \pm 1, \pm 2, \ldots
$$

Так как $\overline{\Psi_{n}^{ \pm}(\lambda)}=\Psi_{n}^{\mp}(\lambda)$ при $\lambda \in \partial \Gamma$, то

$$
\int_{\partial \Gamma} \rho_{0}(\lambda) f(\lambda) \Psi_{n}^{ \pm}(\lambda) d \lambda=0, \quad n=0, \pm 1, \pm 2, \ldots
$$


В силу (1.1) имеем равенство

$$
\int_{\partial \Gamma} \rho_{0}(\lambda) f(\lambda) \Psi_{n}^{ \pm}(\lambda) \lambda^{m} d \lambda=0, \quad m=0,1,2, \ldots,
$$

согласно которому при всех $n, k=0, \pm 1, \pm 2, \ldots$

$$
\int_{\partial \Gamma} \rho_{0}(\lambda) f(\lambda)\left(\Psi_{n}^{ \pm}(\lambda)-\Psi_{k}^{ \pm}(\lambda)\right) \lambda^{m} d \lambda=0 .
$$

Полагая $n=N, k=-N$ и учитывая (1.3), получаем

$$
\int_{\sigma} \lambda^{m} \chi(1, N-1 ; \lambda) \operatorname{Im} f(\lambda+i 0) d \lambda=0, \quad m=0,1,2, \ldots,
$$

откуда следует, что $\operatorname{Im} f(\lambda+i 0)=0$ при $\lambda \in \sigma$.

С другой стороны, выбирая в предпоследнем равенстве $n=2 N, k=0$ и используя (1.3), имеем

$$
\int_{\sigma} \lambda^{m} \chi(1, N-1 ; \lambda) \operatorname{Im}(z(\lambda+i 0) f(\lambda+i 0)) d \lambda=0, \quad m=0,1,2, \ldots
$$

Поэтому $\operatorname{Im}(z(\lambda+i 0) f(\lambda+i 0))=0$ при $\lambda \in \sigma$. Так как $\operatorname{Im} f(\lambda+i 0)=0$, $\operatorname{Im} z(\lambda+i 0) \neq 0$ при $\lambda \in \sigma$, то $\operatorname{Re} f(\lambda+i 0)=0$ при $\lambda \in \sigma$. Следовательно, $f(\lambda)=0$ при $\lambda \in \partial \Gamma$.

Лемма доказана.

Введем теперь “регуляризованные” решения Флоке. С этой целью представим многочлен $\chi(1, N-1 ; \lambda)$ в виде

$$
\chi(1, N-1 ; \lambda)=D^{+}(\lambda) D(\lambda) D^{-}(\lambda)
$$

где корни многочлена $D^{ \pm}(\lambda)$ совпадают с полюсами функции $m^{ \pm}(\lambda)$, а корни многочлена $D(\lambda)$ состоят из двукратных нулей многочлена $\Delta^{2}(\lambda)-1$.

Введем функции $e_{r}^{ \pm}=e_{r}^{ \pm}(\lambda)$, полагая

$$
e_{r}^{ \pm}=D^{ \pm}(\lambda) E_{r}^{ \pm}, \quad r=1, \ldots, N
$$

Тогда при $\lambda \in \partial Г$ имеют место равенства

$$
D^{-}(\lambda) \overline{e_{m}^{ \pm}}=D^{+}(\lambda) e_{m}^{-}, \quad m=1, \ldots, N
$$

Ввиду однородности уравнения (1.1) заметим, что это уравнение имеет решения $\psi_{n}^{ \pm}=\psi_{n}^{ \pm}(\lambda)$, регулярные в $\Gamma$, непрерывные вплоть до $\partial Г$ и представимые в виде

$$
\psi_{n N+j}^{ \pm}=e_{j+1}^{ \pm} z^{ \pm n}, \quad j=0,1, \ldots, N-1 .
$$

Кроме того, в силу (1.7)

$$
\frac{1}{2 \pi i} \int_{\partial \Gamma} \rho(\lambda) \psi_{n}^{+}(\lambda) \psi_{m}^{-}(\lambda) d \lambda=\delta_{n m}, \quad n, m=0, \pm 1, \pm 2, \ldots,
$$

где

$$
\rho(\lambda)=\frac{D(\lambda)}{A\left(z-z^{-1}\right)}
$$




\section{§ 2. Прямая задача рассеяния}

Рассмотрим решения $f_{n}^{ \pm}(\lambda)$ уравнения $(0.1)$, удовлетворяюшие условиям

$$
\lim _{n \rightarrow \pm \infty}\left(f_{n}^{ \pm}(\lambda)-\psi_{n}^{ \pm}(\lambda)\right)=0 .
$$

Как следует из [8], такие решения существуют при $Q_{1}<\infty$ и для них справедливы представления

$$
f_{n}^{ \pm}(\lambda)=\alpha_{n}^{ \pm}\left(\psi_{n}^{ \pm}(\lambda)+\sum_{m=n \pm 1}^{ \pm \infty} K^{ \pm}(n, m) \psi_{n}^{ \pm}(\lambda)\right),
$$

причем имеют место соотношения

$$
\lim _{n \rightarrow \pm \infty} \alpha_{n}^{ \pm}=1, \quad\left|K^{ \pm}(n, m)\right| \leqslant C^{ \pm}(n) \xi^{ \pm}\left(\left[\frac{n+m}{2}\right]\right),
$$

где $\xi^{ \pm}(n)=\sum_{k=n \pm 1}^{ \pm \infty}\left(\left|a_{k}+b_{k}\right|\right),[x]$ - целая часть $x, \pm C^{ \pm}(n)-$ здесь и далее невозрастающая функция от $n$.

Подставляя решения (2.1) в уравнение $(0.1)$, имеем

$$
\begin{gathered}
\frac{\widehat{a}_{n}+a_{n}}{\widehat{a}_{n}}=\frac{\alpha_{n+\theta_{1}^{ \pm}}^{ \pm}}{\alpha_{n-\theta_{-1}^{ \pm}}^{ \pm}}, \quad b_{n}= \pm\left(\widehat{a}_{n} K^{ \pm}(n, n+1)-\widehat{a}_{n-1} K^{ \pm}(n-1, n)\right) \stackrel{\text { def }}{=} \tau_{n}^{ \pm}, \\
\begin{aligned}
& \frac{\left(\widehat{a}_{n}+a_{n}\right)^{2}}{\widehat{a}_{n}}-\widehat{a}_{n}= \pm\left(\widehat{a}_{n+1} K^{ \pm}(n, n+2)-\widehat{a}_{n-1} K^{ \pm}(n-1, n+1)\right) \\
&+\left(\widehat{b}_{n+\theta_{1}^{ \pm}}-\widehat{b}_{n-\theta_{-1}^{ \pm}}-\widehat{b}_{n-\theta_{1}^{ \pm}}\right) K^{ \pm}(n, n+1) \stackrel{\text { def }}{=} \varkappa_{n}^{ \pm}
\end{aligned}
\end{gathered}
$$

где $\theta_{j}^{ \pm}=[(j \pm 1) / 2]$, а при $m>n$ под записью $K^{-}(n, m)$ подразумевается $K^{-}(m, n)$.

Установим теперь связь между решениями $f_{n}^{+}(\lambda)$ и $f_{n}^{-}(\lambda)$. Очевидно, что при $\lambda \in \partial \Gamma$ решениями уравнения (0.1) являются также

$$
\tilde{f}_{n}^{ \pm}(\lambda)=\frac{D^{\mp}(\lambda)}{D^{ \pm}(\lambda)} \overline{f_{n}^{ \pm}(\lambda)}
$$

Используя (1.11) и (2.1), получаем

$$
\tilde{f}_{n}^{ \pm}(\lambda)=\alpha_{n}^{ \pm}\left(\psi_{n}^{\mp}(\lambda)+\sum_{m=n \pm 1}^{ \pm \infty} K^{ \pm}(n, m) \psi_{m}^{\mp}(\lambda)\right), \quad \lambda \in \partial \Gamma .
$$

Пусть множество $\omega=\left\{\lambda_{j}\right\}$ состоит из простых корней многочлена $\Delta^{2}(\lambda)-1$ ${ }^{\prime}{ }^{\prime} \partial \Gamma=\partial \Gamma \backslash \omega$. Тогда при $\lambda \in{ }^{\prime} \partial \Gamma$ пары $\left\{f_{n}^{ \pm}(\lambda), \tilde{f}_{n}^{ \pm}(\lambda)\right\}$ образуют фундаментальные системы решений уравнения (0.1), так как согласно (1.3), (1.9), (1.10), $(2.1),(2.2),(2.4)$ их вронскиан $W\left[f_{n}^{ \pm}, \widetilde{f}_{n}^{ \pm}\right]=\left(\widehat{a}_{n}+a_{n}\right)\left(f_{n}^{ \pm} \widetilde{f}_{n+1}^{ \pm}-f_{n+1}^{ \pm} \widetilde{f}_{n}^{ \pm}\right)$равен $\mp \rho^{-1}(\lambda)$. Следовательно, при $\lambda \in^{\prime} \partial \Gamma$ справедливы разложения

$$
\frac{f_{n}^{\mp}(\lambda)}{a(\lambda)}=\tilde{f}_{n}^{ \pm}(\lambda)+r^{ \pm}(\lambda) f_{n}^{ \pm}(\lambda)
$$


где

$$
\begin{gathered}
r^{+}(\lambda)=\frac{b(\lambda)}{a(\lambda)}, \quad r^{-}(\lambda)=-\left(\frac{D^{+}(\lambda)}{D^{-}(\lambda)}\right)^{2} \frac{\overline{b(\lambda)}}{a(\lambda)}, \\
a(\lambda)=\rho(\lambda) W\left[f_{n}^{-}(\lambda), f_{n}^{+}(\lambda)\right], \quad b(\lambda)=\rho(\lambda) W\left[\widetilde{f}_{n}^{+}(\lambda), f_{n}^{-}(\lambda)\right] .
\end{gathered}
$$

Согласно $(2.1),(2.4)$ и (2.6) функции $a(\lambda)$ и $b(\lambda)$ непрерывны при $\lambda \in \epsilon^{\prime} \partial Г$. Кроме того, из соотношения $z(\lambda-i 0)=\overline{z(\lambda+i 0)}, \lambda \in \sigma$, и из (2.1), (2.4), (2.5) следует, что

$$
\begin{gathered}
a(\lambda-i 0)=\overline{a(\lambda+i 0)}, \quad b(\lambda-i 0)=\overline{b(\lambda+i 0)}, \quad \lambda \in \sigma \backslash \omega, \\
|a(\lambda)|^{2}-\left|\frac{D^{+}(\lambda)}{D^{-}(\lambda)} b(\lambda)\right|^{2}=1, \quad \lambda \in^{\prime} \partial \Gamma .
\end{gathered}
$$

С другой стороны, ввиду соотношений $(2.1),(2.6)$ функция $a(\lambda)$ допускает регулярное продолжение в $\Gamma$, а функция $\rho^{-1}(\lambda) a(\lambda)$ непрерывна вплоть до границы $\partial \Gamma$.

Изучим теперь поведения коэффициентов $a(\lambda), b(\lambda)$ вблизи точек $\lambda_{j}$ множества $\omega$.

Лемма 2.1. Если $\lambda_{0} \in \omega$, то при $Q_{2}<\infty$ коэффициентьи $a(\lambda)$ и $b(\lambda)$ сингулярны или не сингулярны в точке $\lambda_{0}$ одновременно:

$$
\begin{array}{ll}
a(\lambda)=C_{0} \rho(\lambda)+A(\lambda), & \lambda \in \Gamma, \\
b(\lambda)=-C_{0} \frac{D^{-}(\lambda)}{D^{+}(\lambda)} \rho(\lambda)+B(\lambda), & \lambda \in \partial \Gamma,
\end{array}
$$

где $A(\lambda)$ и $B(\lambda)$ непрерывны в точке $\lambda=\lambda_{0}$.

ДокАЗАТЕЛЬСТво. Для определенности примем, что $z\left(\lambda_{0}\right)=1$. Тогда

$$
\begin{aligned}
& f_{0}^{ \pm}(\lambda)-f_{0}^{ \pm}\left(\lambda_{0}\right)=A_{0}^{ \pm}(\lambda)(z-1), \\
& f_{1}^{ \pm}(\lambda)-f_{1}^{ \pm}\left(\lambda_{0}\right)=A_{1}^{ \pm}(\lambda)(z-1) .
\end{aligned}
$$

Докажем, например, первое равенство. Из (1.12), (2.1) следует, что

$$
\begin{aligned}
f_{0}^{+}(\lambda) & -f_{0}^{+}\left(\lambda_{0}\right)=\alpha_{0} \sum_{j=0}^{N-1} e_{j+1}^{+}(\lambda) \sum_{m=0}^{\infty} K^{+}(0, m N+j) z^{m}(\lambda) \\
& -\alpha_{0} \sum_{j=0}^{N-1} e_{j+1}^{+}\left(\lambda_{0}\right) \sum_{m=0}^{\infty} K^{+}(0, m N+j) \\
= & \alpha_{0} \sum_{j=0}^{N-1}\left(e_{j+1}^{+}(\lambda)-e_{j+1}^{+}\left(\lambda_{0}\right)\right) \sum_{m=0}^{\infty} K^{+}(0, m N+j) z^{m}(\lambda) \\
& +\alpha_{0}(z(\lambda)-1) \sum_{j=0}^{N-1} e_{j+1}^{+}(\lambda) \sum_{m=0}^{\infty} K^{+}(0, m N+j) \\
& \times\left(z^{m-1}(\lambda)+z^{m-2}(\lambda)+\cdots+1\right),
\end{aligned}
$$

где $K^{+}(0,0)=1$. 
Согласно $(1.3),(1.10)$ функции $\left(e_{j+1}^{+}(\lambda)-e_{j+1}^{+}\left(\lambda_{0}\right)\right) /(z-1)$ непрерывны в точке $\lambda_{0}$. Кроме этого из условия $Q_{2}<\infty$ и оценки (2.2) вытекает, что функция $\sum_{m=0}^{\infty} K^{+}(0, m N+j)\left(z^{m-1}+z^{m-2}+\cdots+1\right)$ непрерывна вплоть до границы $\partial \Gamma$ плоскости Г. Следовательно, $f_{0}^{+}(\lambda)-f_{0}^{+}\left(\lambda_{0}\right)=A_{0}^{+}(\lambda)(z-1)$, где $A_{0}^{+}(\lambda)$ есть непрерывная функция в точке $\lambda=\lambda_{0}$. Другие соотношения из (2.9) проверяются аналогично.

Далее, так как вронскиан двух решений уравнения (0.1) не зависит (см. [3]) от $n$, то из (2.6) имеем

$$
\begin{aligned}
a(\lambda)= & \rho(\lambda) W\left[f_{0}^{-}\left(\lambda_{0}\right), f_{0}^{+}\left(\lambda_{0}\right)\right]+\rho(\lambda)\left(W\left[f_{0}^{-}(\lambda), f_{0}^{+}(\lambda)\right]-W\left[f_{0}^{-}\left(\lambda_{0}\right), f_{0}^{+}\left(\lambda_{0}\right)\right]\right), \\
b(\lambda)= & \frac{D^{-}(\lambda)}{D^{+}(\lambda)} \rho(\lambda) W\left[\overline{f_{0}^{+}(\lambda)}, f_{0}^{-}\left(\lambda_{0}\right)\right] \\
& \left.+\frac{D^{-}(\lambda)}{D^{+}(\lambda)} \rho(\lambda)\left(W \overline{\left[f_{0}^{+}(\lambda)\right.}, f_{0}^{-}(\lambda)\right]-W\left[\overline{f_{0}^{+}\left(\lambda_{0}\right)}, f_{0}^{-}\left(\lambda_{0}\right)\right]\right) .
\end{aligned}
$$

Полагая $C_{0}=W\left[f_{0}^{-}\left(\lambda_{0}\right), f_{0}^{+}\left(\lambda_{0}\right)\right]$ и учитывая $\overline{f_{0}^{ \pm}\left(\lambda_{0}\right)}=f_{0}^{ \pm}\left(\lambda_{0}\right)$ и равенства $(2.9)$, получаем (2.8). При этом коэффициенты $a(\lambda), b(\lambda)$ сингулярны в точке $\lambda=\lambda_{0}$, если $C_{0} \neq 0$, и не сингулярны, если $C_{0}=0$.

Лемма доказана.

Изучим асимптотическое поведение функции $a(\lambda)$ на бесконечности.

Лемма 2.2. Справедливо соотношение

$$
\lim _{\lambda \rightarrow \infty} a(\lambda)=\alpha_{n}^{+} \alpha_{n}^{-} .
$$

ДокАЗАтЕЛЬСтво. Из (1.3), (1.10), (1.12), (2.1) следует, что

$$
f_{n}^{ \pm}(\lambda)=\alpha_{n}^{ \pm} \psi_{n}^{ \pm}(\lambda)\left(1+O\left(\frac{1}{\lambda}\right)\right), \quad \lambda \rightarrow \infty
$$

Учитывая эту асимптотику в формуле (2.6), получаем (2.10).

Лемма доказана.

В силу леммы 2.2 нули функции $a(\lambda)$ образуют ограниченное множество. Так как $a(\lambda) \neq 0$ при $\lambda \in^{\prime} \partial Г$, то предельньми точками этого множества могут быть лишь простые нули $\lambda_{j} \in \omega$ многочлена $\Delta^{2}(\lambda)-1$. Однако согласно лемме 2.1 это невозможно. Следовательно, нули функции $a(\lambda)$ образуют конечное множество.

$\mathrm{C}$ другой стороны, нули функции $a(\lambda)$, как следует из $(2.6)$, служат собственньми значениями оператора, порожденного левой частью уравнения $(0.1)$ в пространстве $l^{2}(-\infty, \infty)$. В силу (0.2) этот оператор самосопряжен. Поэтому нули функции $a(\lambda)$ вешественны. Таким образом, функция $a(\lambda)$ может иметь только конечное число вещественных нулей $\mu_{k}, k=1,2, \ldots, p$, лежащих вне $\partial Г$. Докажем, что эти нули простые.

Пусть

$$
\begin{gathered}
c_{k}=\frac{f_{n}^{+}\left(\mu_{k}\right)}{f_{n}^{-}\left(\mu_{k}\right)}, \quad k=1, \ldots, p, \\
\left(m_{k}^{ \pm}\right)^{-2}=\sum_{n=-\infty}^{\infty}\left|f_{n}^{ \pm}\left(\mu_{k}\right)\right|^{2} .
\end{gathered}
$$


Лемма 2.3. Нули $\mu_{k}, k=1, \ldots, p$, функиии а $(\lambda)$ простые, и справедливъь равенства

$$
\rho^{-1}\left(\mu_{k}\right) \dot{a}\left(\mu_{k}\right)=-c_{k}^{-1}\left(m_{k}^{+}\right)^{-2}=-c_{k}\left(m_{k}^{-}\right)^{-2}, \quad k=1, \ldots, p,
$$

әде точкой сверху обозначается производная по $\lambda$.

ДокАЗАТЕЛЬСтво. Суммируя соотношения

$$
u_{n} v_{n}=W\left[\dot{u}_{n-1}, v_{n-1}\right]-W\left[\dot{u}_{n}, v_{n}\right]=W\left[\dot{v}_{n-1}, u_{n-1}\right]-W\left[\dot{v}_{n}, u_{n}\right]
$$

при $u_{n}=f_{n}^{+}(\lambda), v_{n}=f_{n}^{-}(\lambda), \lambda=\mu_{k}$, и используя $(2.11)$, получаем

$$
\begin{aligned}
& W\left[\dot{f}_{n}^{+}\left(\mu_{k}\right), f_{n}^{-}\left(\mu_{k}\right)\right]=c_{k}^{ \pm 1} \sum_{m=n+1}^{\infty}\left|f_{m}^{\mp}\left(\mu_{k}\right)\right|^{2}, \\
& W\left[f_{n}^{+}\left(\mu_{k}\right), \dot{f}_{n}^{-}\left(\mu_{k}\right)\right]=c_{k}^{ \pm 1} \sum_{m=-\infty}^{n}\left|f_{m}^{\mp}\left(\mu_{k}\right)\right|^{2} .
\end{aligned}
$$

Складывая эти равенства и учитывая (2.6), (2.12), получаем соотношения (2.13), которые показывают, что нули $\mu_{k}$ являются простыми.

Лемма доказана.

Воспользовавшись (2.13), заметим, что

$$
\left(m_{k}^{+}\right)^{2}\left(m_{k}^{-}\right)^{2}=\left(\rho^{-1}\left(\mu_{k}\right) \dot{a}\left(\mu_{k}\right)\right)^{-2}, \quad k=1, \ldots, p
$$

Выпишем некоторые из установленных свойств в виде условия, которое нам понадобится.

I) Функиии $a(\lambda), b(\lambda)$ непрерывны при $\lambda \in^{\prime} \partial \Gamma$, удовлетворяют соотношениям (2.7), (2.8). Функиия $а(\lambda)$ регулярно продолжима в плоскости $\Gamma$, имеет асимптотику $\lim _{\lambda \rightarrow \infty} a(\lambda)=d>0$ и может иметь простые нули в точках $\mu_{k}, k=1, \ldots, p$. Функция $\rho^{-1}(\lambda) a(\lambda)$ непрерывна вплоть до границы $\partial Г$. Нормировочнье числа $m_{k}^{+}$и $m_{k}^{-}$связань соотношениями (2.14).

Известно [16], что ограниченные при $n \rightarrow \pm \infty$ решения уравнения вида $(0.1)$ называются “волновыми” функииями. Если заданы “потенциалы" возмущения $a_{n}, b_{n}$ и надо определить асимптотическое поведение волновых функций, то мы имеем прямую задачу рассеяния. Обратная же задача рассеяния - нахождение "потенциалов" возмушения $a_{n}, b_{n}$ по заданным асимптотикам "волновых" функций и по невозмущенньм "потенциалам" $\widehat{a}_{n}, \widehat{b}_{n}$. В математических терминах обратная задача рассеяния для уравнения вида (0.1) состоит в восстановлении возмущенного оператора, порождаемого этим уравнением в $l^{2}(-\infty, \infty)$, по асимптотикам “собственных" функций и по соответствующему невозмущенному оператору (cм. [3], [4]).

В силу (2.1), (2.2), (2.5) уравнение (0.1) имеет ограниченные решения при $\lambda \in \sigma$ и $\lambda=\mu_{k}, k=1, \ldots, p$. Кроме того, согласно $(2.5),(2.11),(2.13)$ набор величин $\left\{a(\lambda), b(\lambda), \lambda \in^{\prime} \partial \Gamma ; \mu_{k} ; m_{k}^{ \pm}>0, k=1, \ldots, p\right\}$ полностью определяет асимптотики при $n \rightarrow \pm \infty$ ограниченных решений уравнения (0.1). 
ОПРЕДЕЛЕНИЕ 2.1. Набор величин

$$
\left\{a(\lambda), b(\lambda), \lambda \in^{\prime} \partial \Gamma ; \mu_{k} ; m_{k}^{ \pm}>0, k=1, \ldots, p\right\}
$$

назовем данными рассеяния для уравнения (0.1).

Обратная задача рассеяния для уравнения (0.1) заключается в восстановлении коэффициентов $a_{n}, b_{n}$ этого уравнения по невозмушенным коэффициентам $\widehat{a}_{n}, \widehat{b}_{n}$ и по данным рассеяния.

\section{§3. Вывод основных уравнений}

Выведем для нашей задачи уравнения, связьвающие данные рассеяния с ядрами $K^{ \pm}(n, m)$.

Введем обозначения

$$
\begin{aligned}
M^{ \pm}(n, m) & =\sum_{k=1}^{p}\left(m_{k}^{ \pm}\right)^{2} \psi_{n}^{ \pm}\left(\mu_{k}\right) \psi_{m}^{ \pm}\left(\mu_{k}\right) \\
S^{ \pm}(n, m) & = \pm \frac{1}{2 \pi i} \int_{\partial \Gamma} \rho(\lambda) r^{ \pm}(\lambda) \psi_{n}^{ \pm}(\lambda) \psi_{m}^{ \pm}(\lambda) d \lambda \\
F^{ \pm}(n, m) & =M^{ \pm}(n, m)+S^{ \pm}(n, m) .
\end{aligned}
$$

ЛЕмма 3.1. Имеют место следующие соотношения:

$$
\begin{aligned}
& F^{ \pm}(n, m)+K^{ \pm}(n, m)+\sum_{s=n \pm 1}^{ \pm \infty} K^{ \pm}(n, s) F^{ \pm}(s, m)=0, \quad \pm m \geqslant \pm(n \pm 1), \\
& \left(\alpha_{n}^{ \pm}\right)^{-2}=1+F^{ \pm}(n, n)+\sum_{s=n \pm 1}^{ \pm \infty} K^{ \pm}(n, s) F^{ \pm}(s, n), \quad n=0, \pm 1, \pm 2, \ldots
\end{aligned}
$$

ДокАЗАТЕЛьство. Умножая обе части равенства (2.5) на

$$
\frac{\left(\alpha_{n}^{ \pm}\right)^{-1}}{2 \pi i} \rho(\lambda) \psi_{m}^{ \pm}(\lambda), \quad \pm m \geqslant \pm n
$$

и подставляя вместо $f_{n}^{ \pm}(\lambda), \widetilde{f}_{n}^{ \pm}(\lambda)$ их представления в виде $(2.1),(2.4)$, проведем интегрирование по ӘГ. Используя (1.13), получаем

$$
\begin{aligned}
& \frac{\left(\alpha_{n}^{ \pm}\right)^{-1}}{2 \pi i} \int_{\partial \Gamma} \rho(\lambda) f_{n}^{\mp}(\lambda) a^{-1}(\lambda) \psi_{m}^{ \pm}(\lambda) d \lambda \\
& \quad=\delta_{n m}+\left(1-\delta_{n m}\right) K^{ \pm}(n, m)+S^{ \pm}(n, m)+\sum_{r=n \pm 1}^{ \pm \infty} K^{ \pm}(n, r) S^{ \pm}(r, m) .
\end{aligned}
$$


С другой стороны, применяя теорему о вычетах и учитывая (1.8), (1.10), (1.12), (1.14), а также используя лемму 2.2, имеем

$$
\begin{aligned}
& \frac{\left(\alpha_{n}^{ \pm}\right)^{-1}}{2 \pi i} \int_{\partial \Gamma} \rho(\lambda) f_{n}^{\mp}(\lambda) a^{-1}(\lambda) \psi_{m}^{ \pm}(\lambda) d \lambda \\
& \quad=\left(\alpha_{n}^{ \pm}\right)^{-2} \delta_{n m}-M^{ \pm}(n, m)-\sum_{r=n \pm 1}^{ \pm \infty} K^{ \pm}(n, r) M^{ \pm}(r, m) .
\end{aligned}
$$

Сравнивая первое и последнее равенства, приходим к соотношениям (3.1), (3.2).

\section{Лемма доказана.}

Уравнения (3.1) назовем основными уравнениями типа Марченко.

Используем теперь уравнение (3.1) для уточнения свойств величины $F^{ \pm}(n, m)$. Полагая

$$
\begin{aligned}
e(\lambda ; j) & =\left(e_{1}^{+}, \ldots, e_{j}^{+}, e_{j+1}^{+} z^{-1}, \ldots, e_{N}^{+} z^{-1}\right), \quad j=1, \ldots, N, \\
A_{n m}^{s j} & = \begin{cases}K^{+}(n N+s-1,(n+m) N+j-1), & 1 \leqslant j \leqslant s ; \\
K^{+}(n N+s-1,(n+m-1) N+j-1), & s<j \leqslant N,\end{cases}
\end{aligned}
$$

в силу $(1.12),(2.1),(2.2)$ получаем

$$
\begin{gathered}
f_{n N+j-1}^{+}=\alpha_{n N+j-1} z^{n}\left(e_{j}^{+}+\sum_{m=1}^{\infty}\left(A_{n m}^{j 1}, \ldots, A_{n m}^{j N}\right) e^{*}(\lambda ; j) z^{m}\right), \\
\left|A_{n m}^{s j}\right| \leqslant C_{s j}(n) \xi^{+}\left(n N+\left[\frac{m-1}{2} N\right]\right), \quad j=1, \ldots, N,
\end{gathered}
$$

где $e^{*}(\lambda ; j)$ - транспонированный вектор, $C_{s j}(n)$ - невозрастаюшая функция от $n$. При таких обозначениях уравнение (3.1) для случая “+” видоизменяется следующим образом:

$$
\begin{gathered}
\left(F_{2 n+m}^{s 1}(s), \ldots, F_{2 n+m}^{s N}(s)\right)+\left(A_{n m}^{s 1}, \ldots, A_{n m}^{s N}\right) \\
+\sum_{k=m+1}^{\infty}\left(A_{n, k-m}^{s 1}, \ldots, A_{n, k-m}^{s N}\right) F_{2 n+k}(s)=0 \\
m=1,2, \ldots, \quad s=1,2, \ldots, N
\end{gathered}
$$

где матрица $F_{n}(s)$ определяется формулой

$F_{n}(s)=\sum_{j=1}^{p}\left(m_{j}^{+}\right)^{2} e^{*}\left(\mu_{j} ; s\right) e\left(\mu_{j} ; s\right) z^{n}\left(\mu_{j}\right)+\frac{1}{2 \pi i} \int_{\partial \Gamma} \rho(\lambda) r^{+}(\lambda) e^{*}(\lambda ; s) e(\lambda ; s) z^{n} d \lambda$.

Обозначим $\left\|F_{n}\right\|=\max _{1 \leqslant s \leqslant N} \max _{(i, j)}\left|F_{n}^{i j}(s)\right|$. Из определения $F_{n}(s)$ следует, что при любых $s_{1}$ и $s_{2}, s_{1}<s_{2}$, сушествует такое $l, l=0,1,2$, что

$$
F_{n}^{i j}\left(s_{2}\right)=F_{n+l}^{i j}\left(s_{1}\right) .
$$


Учитывая это и используя (3.3), из последних уравнений находим, что для каждого конечного числа $a$ при $n>a$ верно неравенство

$$
\begin{aligned}
\left\|F_{2 n+m}\right\| \leqslant & C\left(\xi^{+}\left(n N+\left[\frac{m-1}{2} N\right]\right)+\xi^{+}(n N)\right) \\
& +C \sum_{k=m+1}^{\infty} \xi^{+}\left(n N+\left[\frac{k-m-1}{2} N\right]\right)\left\|F_{2 n+k}\right\|,
\end{aligned}
$$

где $C>0$ не зависит от $n$. Полагая $\xi_{0}(n, k)=\xi^{+}(n N+[(k-1) N / 2])+\xi^{+}(n N)$, имеем

$$
\begin{aligned}
& \left\|F_{2 n+m}\right\| \xi^{-1}(n, m) \leqslant C+C \xi_{0}^{-1}(n, m) \\
& \quad \times \sum_{k=m+1}^{\infty} \xi^{+}\left(n N+\left[\frac{k-m-1}{2} N\right]\right) \xi_{0}(n, k)\left(\left\|F_{2 n+k}\right\| \xi_{0}^{-1}(n, k)\right) .
\end{aligned}
$$

Так как последовательности $\gamma_{k}=C \xi_{0}^{-1}(n, m) \xi^{+}(n N+[(k-m-1) N / 2]) \xi_{0}(n, k)$ и $\left\|F_{2 n+k}\right\| \xi_{0}^{-1}(n, k) \gamma_{k}$ суммируемы по $m$ при $m \geqslant k+1$, то в силу разностного аналога леммы Гронуолла (см. [4]) получаем

$$
\left\|F_{2 n+m}\right\| \xi_{0}^{-1}(n, m) \leqslant C \exp \left(\sum_{k=m+1}^{\infty} \gamma_{k}\right)
$$

согласно которому

$$
\left\|F_{2 n+m}\right\| \leqslant C_{1} \xi_{0}(n, m), \quad C_{1}>0 .
$$

Из последнего неравенства следует, что

$$
\left|F_{n}^{i j}(s)\right|<C^{+} \xi^{+}\left(\left[\frac{n N}{2}\right]\right) .
$$

Поэтому если $n=n_{1} N+n_{2}, k=k_{1} N+k_{2}, n_{2}, k_{2}=0,1, \ldots, N-1$, то

$$
\left|F^{+}(n, n+k)\right|<C^{+} \xi^{+}\left(n_{1} N+\left[\frac{k_{1} N}{2}\right]\right)<C^{+} \xi^{+}\left(n+\left[\frac{k}{2}\right]-2 N\right) \text {. }
$$

Таким образом, при $n>a$ для всех $m \geqslant n$ верно неравенство

$$
\left|F^{+}(n, m)\right|<C^{+} \xi^{+}\left(\left[\frac{n+m}{2}\right]-2 N\right) .
$$

Аналогично, при $n<a, a \neq \pm \infty$, для всех $m \leqslant n$ справедливо неравенство

$$
\left|F^{-}(n, m)\right|<C^{-} \xi^{-}\left(\left[\frac{n+m}{2}\right]+2 N\right) \text {. }
$$

Так как $F^{ \pm}(n, m)=F^{ \pm}(m, n)$, то из последних неравенств вытекает следуюшее свойство.

II) При любом $a \neq \pm \infty$ величина $F^{ \pm}(n, m)$ удовлетворяет условию

$$
\sum_{ \pm(n-a)>0} \sup _{ \pm(m-a)>0}\left|n F^{ \pm}(n, m)\right|<\infty .
$$




\section{§4. Исследование основных уравнений}

Предположим, что данные рассеяния удовлетворяют условиям I), II). Построим по данным рассеяния $\left\{a(\lambda), b(\lambda), \lambda \in{ }^{\prime} \partial \Gamma ; \mu_{k} ; m_{k}^{ \pm}>0, k=1, \ldots, p\right\}$ функции $F^{ \pm}(n, m)$ и рассмотрим $(3.1)$ с этими функциями $F^{ \pm}(n, m)$ как уравнения относительно $K^{ \pm}(n, m)$.

ТеОремА 4.1. Пусть для данных рассеяния выполнены условия I), II). Тогда при каждом $n, n=0, \pm 1, \pm 2, \ldots$, уравнение (3.1) имеет единственное решение в $l^{p}(n \pm 1, \pm \infty), p=1,2$.

ДокАЗАТЕЛЬСтво. Воспользовавшись критерием компактности в $l^{p}(n \pm 1, \pm \infty)$

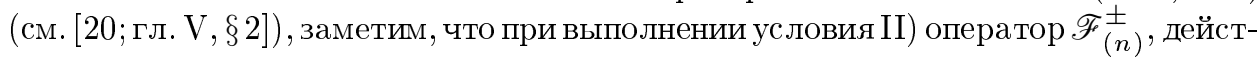
вующий по формуле

$$
\left(\mathscr{F}_{(n)}^{ \pm} h\right)_{m}=\sum_{k=n \pm 1}^{ \pm \infty} F^{ \pm}(k, m) h_{k}, \quad \pm(m-n) \geqslant 1
$$

вполне непрерывен в $l^{p}(n \pm 1, \pm \infty), p=1,2$. В силу альтернативы Фредгольма уравнение (3.1) имеет единственное решение из $l^{p}(n \pm 1, \pm \infty)$, если у однородного уравнения отсутствуют нетривиальные решения из $l^{p}(n \pm 1, \pm \infty)$.

Рассмотрим однородное уравнение

$$
h_{m}^{ \pm}+\sum_{k=n \pm 1}^{ \pm \infty} F^{ \pm}(k, m) h_{k}^{ \pm}=0, \quad \pm(m-n) \geqslant 1 .
$$

В силу вешественности $F^{ \pm}(m, k)$ можно считать, что все $h_{m}^{ \pm}$вещественны. Кроме того, поскольку каждое решение уравнения (3.1) из $l^{1}(n \pm 1, \pm \infty)$ принадлежит и $l^{2}(n \pm 1, \pm \infty)$, достаточно доказать, что (4.1) имеет в $l^{2}(n \pm 1, \pm \infty)$ только тривиальное решение. Пользуясь представлением (1.12) и свойствами класса функций $H_{2}$ (см. [21; гл. $\left.\left.2, \S 4\right]\right)$, заметим, что функция

$$
H^{ \pm}(\lambda)=\sum_{k=n \pm 1}^{ \pm \infty} h_{m}^{ \pm} \psi_{m}^{ \pm}(\lambda)
$$

регулярная в $Г$, имеет почти всюду на $\partial Г$ конечные угловые граничные значения, причем

$$
\frac{1}{2 \pi i} \int_{\partial \Gamma} \rho(\lambda)\left|H^{ \pm}(\lambda)\right|^{2} d \lambda<\infty .
$$

Согласно (1.11), (1.12) при $\lambda \in \partial Г$ имеем

$$
\overline{H^{ \pm}(\lambda)}=\frac{D^{ \pm}(\lambda)}{D^{\mp}(\lambda)} \sum_{m=n \pm 1}^{ \pm \infty} h_{m}^{ \pm} \psi_{m}^{\mp}(\lambda) .
$$

Поэтому из леммы 1.2 вытекает, что

$$
\frac{1}{2 \pi i} \int_{\partial \Gamma} \rho_{1}(\lambda)\left|H^{ \pm}(\lambda)\right|^{2} d \lambda=\sum_{m=n \pm 1}^{ \pm \infty}\left|h_{m}^{ \pm}\right|^{2},
$$


где

$$
\rho_{1}(\lambda)=\frac{\chi(1, N-1 ; \lambda)}{\left(D^{+}(\lambda)\right)^{2} A\left(z-z^{-1}\right)} .
$$

Умножим теперь обе части равенства (4.1) на $h_{m}^{ \pm}$и просуммируем по $m$ в пределах от $n \pm 1$ до $\pm \infty$ :

$$
\sum_{m=n \pm 1}^{ \pm \infty}\left|h_{m}^{ \pm}\right|^{2}+\sum_{m=n \pm 1}^{ \pm \infty} h_{m}^{ \pm} \sum_{k=n \pm 1}^{ \pm \infty} F^{ \pm}(m, k) h_{k}^{ \pm}=0
$$

Пользуясь определениями величин $H^{ \pm}(\lambda), F^{ \pm}(m, k)$ и предпоследним равенством, получаем

$$
\begin{aligned}
& \frac{1}{2 \pi} \int_{\sigma} \widetilde{\rho}_{1}(\lambda)\left(2\left|H^{ \pm}(\lambda+i 0)\right|^{2}+R^{ \pm}(\lambda)\left(H^{ \pm}(\lambda+i 0)\right)^{2}+\overline{R^{ \pm}(\lambda)}\left(\overline{H^{ \pm}(\lambda+i 0)}\right)^{2}\right) d \lambda \\
& \quad+\sum_{j=1}^{p}\left(m_{j}^{ \pm}\right)^{2}\left(H^{ \pm}\left(\mu_{j}\right)\right)^{2}=0
\end{aligned}
$$

где

$$
\widetilde{\rho}_{1}(\lambda)=-i \rho_{1}(\lambda+i 0), \quad R^{ \pm}(\lambda)=\frac{D^{ \pm}(\lambda)}{D^{\mp}(\lambda)} r^{ \pm}(\lambda+i 0), \quad \lambda \in \sigma \backslash \omega .
$$

Как показано в $\S 1, \widehat{\rho}(\lambda)>0$ при $\lambda \in \sigma \backslash \omega$. Поэтому $\widehat{\rho}_{1}(\lambda)>0$ при $\lambda \in \sigma \backslash \omega$, так как $\widehat{\rho}_{1}(\lambda)=\widehat{\rho}(\lambda) /\left(D^{+}(\lambda)\right)^{2}$. Кроме того, так как в силу (2.6), (2.7) $\left|R^{ \pm}(\lambda)\right|<1$ при $\lambda \in \sigma \backslash \omega$, то

$$
\begin{aligned}
& 2\left|H^{ \pm}(\lambda+i 0)\right|^{2}+R^{ \pm}(\lambda)\left(H^{ \pm}(\lambda+i 0)\right)^{2}+\overline{R^{ \pm}(\lambda)}\left(\overline{H^{ \pm}(\lambda+i 0)}\right)^{2} \\
& \geqslant\left|\overline{H^{ \pm}(\lambda+i 0)}+R^{ \pm}(\lambda) H^{ \pm}(\lambda+i 0)\right|^{2},
\end{aligned}
$$

поэтому для выполнения равенства (4.3) необходимо, чтобы $H^{ \pm}(\lambda) \equiv 0$ при $\lambda \in \partial \Gamma$. В силу (4.2) получаем $h_{m}^{ \pm} \equiv 0$, т.е. однородное уравнение (4.1) имеет только тривиальное решение.

Теорема доказана.

В силу доказанной теоремы сушествует ограниченный в $l^{1}(n \pm 1, \pm \infty)$ обратный оператор $\left(I+\mathscr{F}_{(n)}^{ \pm}\right)^{-1}, n=0, \pm 1, \pm 2, \ldots$ Более того, согласно условию II)

$$
\left\|\mathscr{F}_{(n)}^{ \pm}\right\|_{l^{1}(n \pm 1, \pm \infty)} \leqslant \sum_{m=n \pm 1}^{ \pm \infty} \sup _{ \pm(k-n) \geqslant 1}\left|F^{ \pm}(m, k)\right| \rightarrow 0, \quad n \rightarrow \pm \infty
$$

откуда следует, что семейство операторов $\left(I+\mathscr{F}_{(n)}^{ \pm}\right)^{-1}$ равномерно ограничено в $l^{1}(n \pm 1, \pm \infty)$ при $\pm(n-a)>0$ :

$$
\left\|\left(I+\mathscr{F}_{(n)}^{ \pm}\right)^{-1}\right\|_{l^{1}(n \pm 1, \pm \infty)} \leqslant C^{ \pm}(a) .
$$

Тогда из уравнения (3.1) с учетом последнего неравенства имеем

откуда

$$
\sum_{m=n \pm 1}^{ \pm \infty}\left|K^{ \pm}(n, m)\right| \leqslant C^{ \pm}(n) \sum_{m=n \pm 1}^{ \pm \infty}\left|F^{ \pm}(m, n)\right|
$$

$$
\left|K^{ \pm}(n, m)\right| \leqslant\left|F^{ \pm}(n, m)\right|+C^{ \pm}(n) \sup _{ \pm(k-n) \geqslant 1}\left|F^{ \pm}(m, k)\right| .
$$


ЗАмЕчАнИЕ 4.1. Если данные рассеяния удовлетворяют условиям I), II) и $K^{ \pm}(n, m)$ - решение уравнения (3.1), то имеет место неравенство

$$
\left(\alpha_{n}^{ \pm}\right)^{-2} \stackrel{\text { def }}{=} 1+F^{ \pm}(n, n)+\sum_{m=n \pm 1}^{ \pm \infty} K^{ \pm}(n, m) F^{ \pm}(m, n)>0 .
$$

Действительно, если $H_{n}^{ \pm}(\lambda)=\sum_{m=n \pm 1}^{ \pm \infty} K^{ \pm}(n, m) \psi_{m}^{ \pm}(\lambda)$, то

$$
\begin{aligned}
& \left(\alpha_{n}^{ \pm}\right)^{-2}>\frac{1}{2 \pi} \int_{\sigma} \widehat{\rho}_{1}(\lambda) \mid \overline{H_{n}^{ \pm}(\lambda+i 0)}+\overline{\psi_{n}^{ \pm}(\lambda+i 0)} \\
& \quad+\left.R^{ \pm}(\lambda)\left(H_{n}^{ \pm}(\lambda+i 0)+\psi_{n}^{ \pm}(\lambda+i 0)\right)\right|^{2} d \lambda+\sum_{j=1}^{\widetilde{N}}\left(\mu_{j}^{ \pm}\right)^{2}\left|H_{n}^{ \pm}\left(\mu_{j}\right)+\psi_{n}^{ \pm}\left(\mu_{j}\right)\right|^{2}
\end{aligned}
$$

Этот факт доказывается с помощью рассуждений, аналогичных приведенным при доказательстве теоремы 4.1.

\section{§5. Обратная задача рассеяния. Основная теорема}

В $\S 2$ и $\S 3$ были получены необходимые условия I), II) на данные рассеяния в случае $Q_{2}<\infty$. Докажем, что эти условия также достаточны, чтобы по невозмущенным коэффициентам $\widehat{a}_{n}, \widehat{b}_{n}$ и по данным рассеяния можно было однозначно восстановить коэффициенты $a_{n}, b_{n}$, удовлетворяющие требованию $Q_{1}<\infty$.

Итак, пусть условия I), II) выполняются. Тогда уравнение (3.1) имеет единственное решение $K^{ \pm}(n, m)$. Определим величины $b_{n}^{ \pm}=\tau_{n}^{ \pm}, \varkappa_{n}^{ \pm}$и $\alpha_{n}^{ \pm}>0$ по формулам $(2.3),(4.5)$ соответственно. Определим также величины $a_{n}^{+}$и $a_{n}^{-}$из соотношений

$$
\frac{\widehat{a}_{n}+a_{n}^{+}}{\widehat{a}_{n}}=\frac{\alpha_{n+1}^{+}}{\alpha_{n}^{+}}, \quad \frac{\widehat{a}_{n}+a_{n}^{-}}{\widehat{a}_{n}}=\frac{\alpha_{n}^{-}}{\alpha_{n+1}^{-}} .
$$

Положим

$$
f_{n}^{ \pm}(\lambda)=\alpha_{n}^{ \pm}\left(\psi_{n}^{ \pm}(\lambda)+\sum_{m=n \pm 1}^{ \pm \infty} K^{ \pm}(n, m) \psi_{m}^{ \pm}(\lambda)\right)
$$

ТЕОРема 5.1. Величина $f_{n}^{ \pm}=f_{n}^{ \pm}(\lambda)$ удовлетворяет уравнению

$$
\begin{aligned}
& \widehat{a}_{n-[(1 \pm 1) / 2]} \frac{\alpha_{n}^{ \pm}}{\alpha_{n \mp 1}^{ \pm}} f_{n \mp 1}^{ \pm}+\left(\widehat{b}_{n}+b_{n}^{ \pm}\right) f_{n}^{ \pm} \\
& \quad+\frac{\alpha_{n}^{ \pm}}{\alpha_{n \pm 1}^{ \pm}}\left(\widehat{a}_{n+[(-1 \pm 1) / 2]}+\varkappa_{n+[(-1 \pm 1) / 2]}^{ \pm}\right) f_{n \pm 1}^{ \pm}=\lambda f_{n}^{ \pm}, \quad n=0, \pm 1, \pm 2, \ldots,
\end{aligned}
$$

əде $[x]-$ целая часть $x$. 
ДокАЗАТЕльство. Докажем теорему для случая “+”. Так как $K^{+}(n, m)$ удовлетворяет оценке (4.4), то достаточно проверить, что

$$
\begin{aligned}
& \widehat{a}_{n-1} K^{+}(n-1, m)+\left(\widehat{b}_{n}+b_{n}^{+}\right) K^{+}(n, m)+\left(\widehat{a}_{n}+\varkappa_{n}^{+}\right) K^{+}(n+1, m) \\
& \quad=\widehat{a}_{m-1} K^{+}(n, m-1)+\widehat{b}_{m} K^{+}(n, m)+\widehat{a}_{m} K^{+}(n, m+1), \quad m \geqslant n+2 .
\end{aligned}
$$

Рассмотрим разностное выражение

$(\widehat{H} y)_{n m}=\widehat{a}_{n-1} y_{n-1, m}+\widehat{a}_{n} y_{n+1, m}+\widehat{b}_{n} y_{n m}-\widehat{a}_{m-1} y_{n, m-1}-\widehat{a}_{m} y_{n, m+1}-\widehat{b}_{m} y_{n m}$.

Ясно, что $\widehat{H} F^{+}(n, m)=0$. Тогда из (3.1) находим, что

$$
\left(\widehat{H} K^{+}\right)_{n m}+\left(\widehat{H}\left(\sum_{l=n+1}^{\infty} K^{+}(n, l) F^{+}(l, m)\right)\right)_{n m}=0 .
$$

Преобразуя второе слагаемое, имеем

$$
\begin{aligned}
& \left(\widehat{H} K^{+}\right)_{n m}+\sum_{l=n+2}^{\infty}\left(\widehat{H} K^{+}\right)_{n l} F^{+}(l, m) \\
& \quad=b_{n}^{+} F^{+}(n, m)+\varkappa_{n}^{+} F^{+}(n+1, m)+b_{n}^{+} K^{+}(n, n+1) F^{+}(n+1, m) .
\end{aligned}
$$

Определим теперь разностное выражение $H$, полагая

$$
(H y)_{n m}=(\widehat{H} y)_{n m}+b_{n}^{+} y_{n m}+\varkappa_{n}^{+} y_{n+1, m} .
$$

Согласно уравнению (3.1)

$$
\begin{aligned}
& b_{n}^{+} F^{+}(n, m) \\
& \quad=-b_{n}^{+}\left(K^{+}(n, m)+K^{+}(n, n+1) F^{+}(n+1, m)+\sum_{l=n+2}^{\infty} K^{+}(n, l) F^{+}(m, l)\right), \\
& \varkappa_{n}^{+} F^{+}(n+1, m)=-\varkappa_{n}^{+}\left(K^{+}(n+1, m)+\sum_{l=n+2}^{\infty} K^{+}(n+1, l) F^{+}(l, m)\right) .
\end{aligned}
$$

Три последних тождества приводят нас к соотношению

$$
\left(H K^{+}\right)_{n m}+\sum_{l=n+2}^{\infty}\left(H K^{+}\right)_{n l} F^{+}(l, m)=0 .
$$

Тогда $\left(H K^{+}\right)_{n m} \equiv 0$, так как в силу теоремы 4.1 последнее уравнение имеет лишш тривиальное решение. Следовательно, имеет место равенство (5.2). Случай “-” рассматривается аналогично.

Теорема доказана.

Наша ближайшая цель состоит теперь в том, чтобы установить равенства $a_{n}^{+}=a_{n}^{-}, b_{n}^{+}=b_{n}^{-}$. 
Определим величину $G(n, m)$ :

$$
G(n, m)=R^{+}(n, m)+K^{+}(n, m)+\sum_{l=n+1}^{\infty} K^{+}(n, l) R^{+}(l, m),
$$

где $K^{+}(n, m) \equiv 0$ при $m \leqslant n$. Ясно, что при каждом $n$ величина $G^{+}(n, \cdot)$ принадлежит пространству $l^{2}(-\infty, \infty)$. Из основного уравнения (3.1) и формулы (5.1) имеем

$$
G(n, m)= \begin{cases}-\left(\alpha_{n}^{+}\right)^{-1} \sum_{j=1}^{p}\left(m_{j}^{+}\right)^{2} f_{n}^{+}\left(\mu_{j}\right) \psi_{m}^{+}\left(\mu_{j}\right), & m \geqslant n+1 ; \\ R^{+}(n, m)+\sum_{l=n+1}^{\infty} K^{+}(n, l) R^{+}(l, m), & m \leqslant n .\end{cases}
$$

Умножим обе части равенства $(5.3)$ на $\psi_{m}^{-}(\lambda), \lambda \in \partial \Gamma$, и просуммируем по $m$ в пределах от $-\infty$ до $+\infty$ :

$$
\begin{aligned}
& \sum_{m=-\infty}^{\infty} R^{+}(n, m) \psi_{m}^{-}(\lambda)+\sum_{m=n+1}^{\infty} K^{+}(n, m) \psi_{m}^{-}(\lambda) \\
& \quad+\sum_{l=n+1}^{\infty} K^{+}(n, l) \sum_{m=-\infty}^{\infty} R^{+}(l, m) \psi_{m}^{-}(\lambda)=\sum_{m=-\infty}^{\infty} G(n, m) \psi_{m}^{-}(\lambda),
\end{aligned}
$$

где сходимости рядов (кроме второго) понимаются в смысле метрики пространства $L_{2}\left(\partial \Gamma ; \rho_{0}(\lambda)\right)$. Согласно определению величины $R^{+}(n, m)($ см. $\S 3)$ и лемме 1.2 получаем

$$
\sum_{m=-\infty}^{\infty} R^{+}(n, m) \psi_{m}^{-}(\lambda)=r^{+}(\lambda) \psi_{m}^{+}(\lambda), \quad \lambda \in \partial \Gamma
$$

Прибавляя к обеим частям предпоследнего равенства $\psi_{n}^{-}(\lambda)$ и умножая на $\alpha_{n}^{+}$, с учетом последней формулы имеем

$$
r^{+}(\lambda) f_{n}^{+}(\lambda)+\widetilde{f}_{n}^{+}(\lambda)=\alpha_{n}^{+}\left(\psi_{n}^{-}(\lambda)+\sum_{m=-\infty}^{\infty} G(n, m) \psi_{m}^{-}(\lambda)\right),
$$

где

$$
\tilde{f}_{n}^{+}(\lambda)=\alpha_{n}^{+}\left(\psi_{n}^{-}(\lambda)+\sum_{m=n+1}^{\infty} K^{+}(n, m) \psi_{m}^{-}(\lambda)\right) .
$$

Умножая обе части последнего равенства на $a(\lambda)$ и обозначая

$$
g_{n}^{-}(\lambda)=a(\lambda) \alpha_{n}^{+}\left(\psi_{n}^{-}(\lambda)+\sum_{m=-\infty}^{\infty} G(n, m) \psi_{m}^{-}(\lambda)\right)
$$

находим, что

$$
g_{n}^{-}(\lambda)=a(\lambda) \tilde{f}_{n}^{+}(\lambda)+b(\lambda) f_{n}^{+}(\lambda), \quad \lambda \in^{\prime} \partial \Gamma
$$


ЛЕмма 5.1. Функиия $g_{n}^{-}(\lambda)$ допускает регулярное продолжение в плоскости Г и непрерывна вплоть до границы ӘГ этой плоскости.

ДокАЗАТЕЛьство. Сначала докажем, что $g_{n}^{-}(\lambda)$ непрерывна при $\lambda \in \partial \Gamma$. Согласно условию I) коэффициенты $a(\lambda)$ и $b(\lambda)$ непрерывны при $\lambda \in^{\prime} \partial \Gamma$. Кроме того, из условия II) и из $(4.4),(5.1)$ следует, что функции $f_{n}^{+}(\lambda)$ и $\widetilde{f}_{n}^{+}(\lambda)$ непрерывны при $\lambda \in \partial \Gamma$. Тогда из (5.6) вытекает непрерывность функции $g_{n}^{-}(\lambda)$ при $\lambda \in{ }^{\prime} \partial \Gamma$.

С другой стороны, из (5.6) имеем

$$
g_{n}^{-}(\lambda)=\frac{D^{-}(\lambda)}{D^{+}(\lambda)} \rho^{-1}(\lambda) a(\lambda) \rho(\lambda)\left(\overline{f_{n}^{+}(\lambda)}-f_{n}^{+}(\lambda)\right)+\left(\frac{D^{-}(\lambda)}{D^{+}(\lambda)} a(\lambda)+b(\lambda)\right) f_{n}^{+}(\lambda)
$$

Используя условие II) и оценку (4.4), легко убедиться, что $\rho(\lambda)\left(\overline{f_{n}^{+}(\lambda)}-f_{n}^{+}(\lambda)\right)-$ непрерывная в точках $\lambda_{i}, \lambda_{j} \in \omega$, функция. Кроме этого в силу условия I) функции $\rho^{-1}(\lambda) a(\lambda)$ и $D^{-}(\lambda) / D^{+}(\lambda) a(\lambda)+b(\lambda)$ также непрерывны в точках $\lambda_{i}, \lambda_{j} \in \omega$. Следовательно, функция $g_{n}^{-}(\lambda)$ непрерывна в точках $\lambda_{i}, \lambda_{j} \in \omega$. Тем самым непрерывность функции $g_{n}^{-}(\lambda)$ при $\lambda \in \partial \Gamma$ доказана.

Представим теперь $g_{n}^{-}(\lambda)$ в виде

$$
g_{n}^{-}(\lambda)=a(\lambda) \alpha_{n}^{+}\left(\psi_{n}^{-}(\lambda)+\sum_{m=-\infty}^{n} G(n, m) \psi_{m}^{-}(\lambda)+\sum_{m=n+1}^{\infty} G(n, m) \psi_{m}^{-}(\lambda)\right) .
$$

В силу $(1.2),(1.3),(1.10),(1.12)$ функция $\sum_{m=-\infty}^{n} G(n, m) \psi_{m}^{-}(\lambda)$ допускает регулярное продолжение в Г. Кроме того, из (5.4) вытекает, что

$$
\sum_{m=n+1}^{\infty} G(n, m) \psi_{m}^{-}(\lambda)=-\left(\alpha_{n}^{+}\right)^{-1} \sum_{j=1}^{p}\left(m_{j}^{+}\right)^{2} f_{n}^{+}\left(\mu_{j}\right) \sum_{m=n+1}^{\infty} \psi_{m}^{+}\left(\mu_{j}\right) \psi_{m}^{-}(\lambda)
$$

Пусть $n=n_{1} N+k-1, k=1, \ldots, N$. Тогда из (1.12) следует, что

$$
\begin{aligned}
\sum_{m=n+1}^{\infty} \psi_{m}^{+}\left(\mu_{j}\right) \psi_{m}^{-}(\lambda)= & \left(\sum_{r=1}^{k} e_{r}^{+}\left(\mu_{j}\right) e_{r}^{-}(\lambda)\right) \sum_{m=n_{1}+1}^{\infty}\left(\frac{z\left(\mu_{j}\right)}{z(\lambda)}\right)^{m} \\
& +\left(\sum_{r=k+1}^{N} e_{r}^{+}\left(\mu_{j}\right) e_{r}^{-}(\lambda)\right) \sum_{m=n_{1}}^{\infty}\left(\frac{z\left(\mu_{j}\right)}{z(\lambda)}\right)^{m},
\end{aligned}
$$

откуда

$$
\begin{aligned}
\sum_{m=n+1}^{\infty} \psi_{m}^{+}\left(\mu_{j}\right) \psi_{m}^{-}(\lambda)= & z^{-n_{1}}(\lambda) \frac{z^{n_{1}+1}\left(\mu_{j}\right)}{z(\lambda)-z\left(\mu_{j}\right)} \sum_{r=1}^{k} e_{r}^{+}\left(\mu_{j}\right) e_{r}^{-}(\lambda) \\
& +z^{1-n_{1}}(\lambda) \frac{z^{n_{1}}\left(\mu_{j}\right)}{z(\lambda)-z\left(\mu_{j}\right)} \sum_{r=k+1}^{N} e_{r}^{+}\left(\mu_{j}\right) e_{r}^{-}(\lambda) .
\end{aligned}
$$

Последняя формула показывает, что функция $\sum_{m=n+1}^{\infty} \psi_{m}^{+}\left(\mu_{j}\right) \psi_{m}^{-}(\lambda)$ и тем самьм функция $\sum_{m=n+1}^{\infty} G(n, m) \psi_{m}^{-}(\lambda)$ допускает регулярное продолжение в область $\Gamma \backslash\left\{\mu_{j}\right\}_{j=1}^{p}$. Вместе с тем из условия I) и из (1.6), (1.10), (1.14), (5.8), (5.9) 
вытекает, что функция $a(\lambda) \sum_{m=n+1}^{\infty} G(n, m) \psi_{m}^{-}(\lambda)$ регулярна в $\Gamma$, а в точках $\mu_{j}$, $j=1, \ldots, p$, имеют место равенства

$$
\left.\left(a(\lambda) \sum_{m=n+1}^{\infty} G(n, m) \psi_{m}^{-}(\lambda)\right)\right|_{\lambda=\mu_{j}}=-\left(\alpha_{n}^{+}\right)^{-1} \rho^{-1}\left(\mu_{j}\right) \dot{a}\left(\mu_{j}\right)\left(m_{j}^{+}\right)^{2} f_{n}^{+}\left(\mu_{j}\right) .
$$

Тогда из (2.14) и (5.7) получим

$$
g_{n}^{-}\left(\mu_{j}\right)=-\left(m_{j}^{-}\right)^{-2} \frac{\rho\left(\mu_{j}\right) f_{n}^{+}\left(\mu_{j}\right)}{\dot{a}\left(\mu_{j}\right)}, \quad j=1, \ldots, p .
$$

Далее, известно, что (см. $[21 ;$ гл. $2, \S \S 4,5])$ функция вида $G(z)=\sum_{n=0}^{\infty} C_{n} z^{n}$, где $C_{n} \in l^{2}(0, \infty)$, принадлежит классу $H_{2}$, обладает конечными угловыми значениями почти всюду на окружности $|z|=1$ и представима своим интегралом Кошш в замкнутом круге $|z| \leqslant 1$. В силу конформных свойств функции $z=z(\lambda)$, упомянутых в $\S 1$, функция $G(z(\lambda))$ обладает конечными угловыми значениями почти всюду на $\partial \Gamma$.

С другой стороны, для любой точки $\lambda_{0} \in \sigma$ некоторый полукруг

$$
U^{ \pm}=\left\{\lambda:\left|\lambda-\lambda_{0}\right| \leqslant r, \pm \operatorname{Im} \lambda \geqslant 0\right\}
$$

конформно отображается функцией $z=z(\lambda)$ на область $\widehat{U}^{ \pm}$, являющуюся частью круга $|z| \leqslant 1$. Так как функция $G(z)$ представима в области $\widehat{U}^{ \pm}$своим интегралом Кошш, то она принадлежит $[21 ;$ гл. $3, \S 7]$ классу $E_{1}$ в области $\widehat{U}^{ \pm}$. Отсюда следует, что функция

$$
G(z(\lambda)) z^{\prime}(\lambda)=2 H(z(\lambda)) \frac{\Delta^{\prime}(\lambda) z(\lambda)}{z(\lambda)-z^{-1}(\lambda)}
$$

принадлежит классу $E_{1}$ в области $U^{ \pm}$. Тогда верна принадлежность функции $\rho(\lambda) G(z(\lambda))$ классу $E_{1}$ в области $U^{ \pm}$, так как в ней функция $D(\lambda) /\left(\Delta^{\prime}(\lambda) z(\lambda)\right)$ ограничена.

В силу (1.12) и непрерьвности функций $e_{j}^{-}(\lambda), j=1, \ldots, p$, функция

$$
\rho(\lambda) \sum_{m=-\infty}^{n} G(n, m) \psi_{m}^{-}(\lambda)
$$

принадлежит классу $E_{1}$ в полукругах $U^{ \pm}$. Кроме этого из (5.8), (5.9) следует непрерывность функции $\sum_{m=n+1}^{\infty} G(n, m) \psi_{m}^{-}(\lambda)$ в тех же полукругах. Следовательно, $\rho(\lambda) \sum_{m=n+1}^{\infty} G(n, m) \psi_{m}^{-}(\lambda)$ принадлежит классу $E_{1}$ в $U^{ \pm}$. Используя $(5.7)$ и принимая во внимание, что функция $\rho^{-1}(\lambda) \alpha(\lambda)$ непрерьвна вплоть до $\partial Г$, получаем принадлежность функции $g_{n}^{-}(\lambda)$ к классу $E_{1}$ в области $U^{ \pm}$. Следовательно, функция $g_{n}^{-}(\lambda)$ в полукругах $U^{ \pm}$представима $[21 ;$ гл. $3, \S 7]$ своим интегралом Коши. Как отмечалось выше, функция $g_{n}^{-}(\lambda)$ непрерывна при $\lambda \in \partial \Gamma$. Тогда она непрерывна на границе полукруга $U^{ \pm}$. Поэтому [21; гл. $\left.3, \S 4\right]$ функция $g_{n}^{-}(\lambda)$ непрерывна на полукруге $U^{+}$. Так как $\lambda_{0}-$ любая точка из $\sigma$, то $g_{n}^{-}(\lambda)$ непрерьвна вплоть до

Лемма доказана.

Мы теперь подготовлены к тому, чтобы доказать основную теорему. 
Теорема 5.2 (основная). 1) Пусть $Q_{2}<\infty$. Тогда данные рассеяния уравнения вида (0.1) удовлетворяют условиям I), II).

2) По коэффичиентам $\widehat{a}_{n}, \widehat{b}_{n}$ и по данным рассеяния, для которых выполненьи условия I), II), мохсно однозначно восстановить уравнения вида $(0.1)$, для которого коэффициенты $a_{n}, b_{n}$ удовлетворяют условию $Q_{1}<\infty$.

ДокАЗАТЕЛЬство. Первое утверждение теоремы доказано в $\S \S 2,3$. Покажем справедливость второго утверждения.

Из (1.3), (1.10), (1.12), (5.9) следует, что

$$
\sum_{m=n+1}^{\infty} \psi_{m}^{+}\left(\mu_{j}\right) \psi_{m}^{-}(\lambda)=-\psi_{n}^{+}\left(\mu_{j}\right) \psi_{n}^{-}(\lambda)\left(1+O\left(\frac{1}{\lambda}\right)\right), \quad \lambda \rightarrow \infty
$$

Тогда в силу условия I) и формул (5.7), (5.8) получим

$g_{n}^{-}(\lambda)=d \alpha_{n}^{+}\left(1+G(n, n)+\sum_{j=1}^{p}\left(\alpha_{n}^{+}\right)^{-2} f_{n}^{+}\left(\mu_{j}\right)\left(m_{j}^{+}\right)^{2} \psi_{n}^{+}\left(\mu_{j}\right)\right) \psi_{n}^{-}(\lambda)\left(1+O\left(\frac{1}{\lambda}\right)\right)$

С другой стороны, согласно (5.1), (5.4) имеем

$$
\begin{aligned}
1+ & G(n, n)+\sum_{j=1}^{p}\left(\alpha_{n}^{+}\right)^{-1} f_{n}^{+}\left(\mu_{j}\right)\left(m_{j}^{+}\right)^{2} \psi_{n}^{+}\left(\mu_{j}\right) \\
= & +R^{+}(n, n)+\sum_{l=n+1}^{\infty} K^{+}(n, l) R^{+}(l, n)+M^{+}(n, n) \\
& +\sum_{l=n+1}^{\infty} K^{+}(n, l) M^{+}(l, n) \\
= & +F^{+}(n, n)+\sum_{l=n+1}^{\infty} K^{+}(n, l) F^{+}(l, n)=\left(\alpha_{n}^{+}\right)^{-2}
\end{aligned}
$$

где мы учли равенство (4.5). Таким образом,

$$
g_{n}^{-}(\lambda)=\frac{d}{\alpha_{n}^{+}} \psi_{n}^{-}(\lambda)\left(1+O\left(\frac{1}{\lambda}\right)\right), \quad \lambda \rightarrow \infty
$$

Из леммы (5.1) следует, что $g_{n}^{-}(\lambda)$ принадлежит пространству $L_{2}\left(\partial \Gamma ; \rho_{0}(\lambda)\right)$. Разлагая функцию $g_{n}^{-}(\lambda) / D^{-}(\lambda)$ по базису $\left\{\Psi_{n}^{-}(\lambda)\right\}_{n=-\infty}^{\infty}$ и используя теорему о вычетах, с учетом (5.11) заключаем, что

$$
g_{n}^{-}(\lambda)=\frac{d}{\alpha_{n}^{+}}\left(\psi_{n}^{-}(\lambda)+\sum_{m=-\infty}^{n-1} B(n, m) \psi_{m}^{-}(\lambda)\right)
$$

где $B(n, \cdot) \in l^{2}(-\infty, n-1)$. 
Далее, переходя в равенстве (5.6) к сопряженным выражениям и полагая

$$
\widetilde{g}_{n}^{-}(\lambda)=\frac{D^{-}(\lambda)}{D^{+}(\lambda)} \overline{g_{n}^{-}(\lambda)}=\frac{d}{\alpha_{n}^{+}}\left(\psi_{n}^{+}(\lambda)+\sum_{m=-\infty}^{n-1} B(n, m) \psi_{m}^{+}(\lambda)\right),
$$

получаем

$$
\widetilde{g}_{n}^{-}(\lambda)=\left(\frac{D^{-}(\lambda)}{D^{+}(\lambda)}\right)^{2} \overline{b(\lambda)} \widetilde{f}_{n}^{+}(\lambda)+\overline{a(\lambda)} f_{n}^{+}(\lambda) .
$$

Решая (5.6) и (5.13) как систему уравнений с неизвестными $f_{n}^{+}(\lambda)$ и $\tilde{f}_{n}^{+}(\lambda)$ и принимая во внимание $(2.6),(2.7)$, находим, что

$$
\frac{f_{n}^{+}(\lambda)}{a(\lambda)}=\tilde{g}_{n}^{-}(\lambda)+r^{-}(\lambda) g_{n}^{-}(\lambda), \quad \lambda \in^{\prime} \partial \Gamma .
$$

Из этого соотношения, как это было сделано в $\S 3$, используя (5.10), (5.12), (5.13), получаем, что

$$
\begin{gathered}
F^{-}(n, m)+B(n, m)+\sum_{l=-\infty}^{n-1} B(n, l) F^{-}(l, m)=0, \quad m \leqslant n-1, \\
\left(\frac{\alpha_{n}^{+}}{d}\right)^{2}=1+F^{-}(n, n)+\sum_{l=-\infty}^{n-1} B(n, l) F^{-}(l, n) .
\end{gathered}
$$

Так как основное уравнение (3.1) для случая “- " имеет единственное решение из $l^{2}(-\infty, n-1)$, то из предпоследнего равенства следует, что $B(n, m)=K^{-}(n, m)$.

Тогда из (4.5) получим $\left(\alpha_{n}^{+} / d\right)^{2}=\left(\alpha_{n}^{-}\right)^{-2}$. Кроме того, в силу (5.11) имеем

$$
g_{n}^{-}(\lambda)=\alpha_{n}^{-}\left(\psi_{n}^{-}(\lambda)+\sum_{m=-\infty}^{n-1} K^{-}(n, m) \psi_{m}^{-}(\lambda)\right)=f_{n}^{-}(\lambda) .
$$

Теперь из (5.6) и (5.14) и из леммы 5.1 вытекает, что $f_{n}^{-}=f_{n}^{-}(\lambda)$ удовлетворяет уравнению

$$
\widehat{a}_{n-1} \frac{\alpha_{n}^{+}}{\alpha_{n-1}^{+}} f_{n-1}^{-}+\left(\widehat{b}_{n}+b_{n}^{+}\right) f_{n}^{-}+\frac{\alpha_{n}^{+}}{\alpha_{n+1}^{+}}\left(\widehat{a}_{n}+\varkappa_{n}^{+}\right) f_{n+1}^{-}=\lambda f_{n}^{-} .
$$

Подставляя в это уравнение вместо $f_{n}^{-}$его представление (5.1), приравнивая коэффициенты при $\psi_{m}^{-}(\lambda)$ и учитывая $(2.3)$, получаем

$$
\frac{\alpha_{n}^{-}}{\alpha_{n+1}^{+}}=\frac{\widehat{a}_{n}+\varkappa_{n}^{-}}{\widehat{a}_{n}}=\frac{\widehat{a}_{n}+\varkappa_{n}^{+}}{\widehat{a}_{n}}=\frac{\alpha_{n+1}^{+}}{\alpha_{n}^{+}}, \quad b_{n}^{+}=b_{n}^{-} .
$$

Таким образом, если мы решим обратную задачу, то формулы $(2.3),(3.1),(3.2)$ приведут нас к одному и тому же уравнению

$$
\left(\widehat{a}_{n-1}+a_{n-1}\right) y_{n-1}+\left(\widehat{b}_{n}+b_{n}\right) y_{n}+\left(\widehat{a}_{n}+a_{n}\right) y_{n+1}=\lambda y_{n}, \quad n=0, \pm 1, \pm 2, \ldots
$$


где $a_{n}=a_{n}^{+}=a_{n}^{-}, b_{n}=b_{n}^{+}=b_{n}^{-}$. Согласно формулам (2.3), условию II) и оценке (4.4) коэффициенты $a_{n}, b_{n}$ удовлетворяют условию

$$
Q_{1}=\sum_{n=-\infty}^{\infty}|n|\left(\left|a_{n}\right|+\left|b_{n}\right|\right)<\infty
$$

Остается еще проверить справедливость формул

$$
\left(m_{k}^{ \pm}\right)^{-2}=\sum_{n=-\infty}^{\infty}\left|f_{n}^{ \pm}\left(\mu_{k}\right)\right|^{2}, \quad k=1, \ldots, p .
$$

Так как $a_{n}^{+}=a_{n}^{-}, b_{n}^{+}=b_{n}^{-}$, то для вычисления $\sum_{n=-\infty}^{\infty}\left|f_{n}^{ \pm}\left(\mu_{k}\right)\right|^{2}$ можно использовать формулы (2.13), в которых согласно (5.10) следует положить

$$
c_{k}=-\left(m_{k}^{-}\right)^{2} \rho^{-1}\left(\mu_{k}\right) \dot{a}\left(\mu_{k}\right), \quad k=1, \ldots, p .
$$

Отсюда следует и справедливость равенств (5.15).

Теорема доказана.

ЗАмечание 5.1. Как показано в [8], при $N=2$ уравнение (0.1) эквивалентно разностному аналогу одномерной системы Дирака. Следовательно, полученные результаты распространяются на случай системы разностных уравнений Дирака.

\section{Список литературы}

1. Тарнопольский В. Г. Задача рассеяния для разностного уравнения // Докл. АН СССР. 1961. T. 136. № 4. C. 779-782.

2. Case K. M., Kac M. A discrete version of the inverse scattering problem // J. Math. Phys. 1973. V. 14. № 5. P. 594-603.

3. Гусейнов Г. ШІ. Обратная задача теории рассеяния для разностного уравнения второго порядка на всей оси // Докл. АН СССР. 1976. Т. 231. № 5. С. 1045-1048.

4. Гусейнов Г. ШШ. Обратные задачи теории рассеяния для самосопряженных разностных операторов второго порядка. // Дис. ... канд. физ.-матем. наук. М.: МГУ, 1976.

5. Никишин E. M. Разностный оператор Штурма-Лиувилля и некоторые вопросы теории функций // Труды сем. им. И.Г. Петровского. Т. 10. М.: Изд-во МГУ, 1984. С. 3-77.

6. Аптекарев А.И., Никишин Е. М. Задача рассеяния для дискретного оператор Штурма-Лиувилля // Матем. сб. 1983. Т. 121. №3. С. 327-358.

7. Гусейнов И. М., Ханмамедов Аг. Х. Асимптотика при $t \rightarrow \infty$ задачи Коши для цепочки Тоды с начальньми данньми типа ступеньки // ТМФ. 1999. Т. 119. № 3. С. 429-440.

8. Ханмамедов $A$ г. X. Операторы преобразования для возмущенного разностного уравнения Хилла и их одно приложение // Сиб. матем. журн. 2003. Т. 44. № 4. С. 926-937.

9. Geronimo J., Van Assche $W$. Orthogonal polynomials with asymptotically periodic recurrence coefficients // J. Approx. Theory. 1986. V. 46. P. 251-283.

10. Teschl $G$. Jacobi operators and completely integrable nonlinear lattices. Providence, RI: Amer. Math. Soc., 2000. (Math. Surveys Monographs. V. 72.)

11. Bazargan J., Egorova I. Jacobi operator withs step-like asymptotically periodic coefficients // Mat. Fiz. Anal. Geom. 2003. V. 10. № 3. P. 425-442.

12. Khanmamedov Ag. Kh. On the theory of inverse scattering problems for a system of a difference equations // Trans. Acad. Sci. Azerb. Ser. Phys.-Tech. Math. Sci. 2000. V. 20. № 4, Math. Mech. P. 132-135.

13. Newton $R$. G. Inverse scattering by a local impurity in a periodic potential in one dimension // J. Math. Phys. 1983. V. 24. P. 2152-2162. 
14. Фирсова H. E. Прямая и обратная задача рассеяния для одномерного возмущенного оператора Хилла // Матем. сб. 1986. Т. 130 (172). №3(7). С. 349-385.

15. Марченко B. А. Операторы Штурма-Лиувилля и их приложения. Киев: Наукова думка, 1977.

16. Тода М. Теория нелинейных решеток. М.: Мир, 1984.

17. Ханмамедов $A$ г. X. К спектральной теории разностного уравнения с периодическими коэффициентами // Вестн. БГУ. Сер. физ.-матем. наук. 2001. № 1. С. 124-130.

18. Ханмамедов Аг. Х. О конформности отображения, порождаемого функцией Блоха разностного уравнения Хилла // Вестн. БГУ. Сер. физ.-матем. наук. 2004. № 1. С. 115-121.

19. Колмогоров A.H., Фомин С.В. Элементы теории функций и функционального анализа. М.: Наука, 1989.

20. Люстерник Л.А., Соболев В.И. Элементы функционального анализа. М.: Наука, 1965.

21. Привалов И. И. Граничные свойства аналитических функций. М.: ГИТТЛ, 1950.

Бакинский государственньй университет,

Азербайджан
Поступила в редакцию 12.05 .2004 и 04.10 .2004 